\title{
The epistemic goal of a concept: accounting for the rationality of semantic change and variation
}

\author{
Ingo Brigandt \\ Department of Philosophy \\ University of Alberta \\ 2-40 Assiniboia Hall \\ Edmonton, AB T6G 2E7 \\ Canada \\ Email: brigandt@ualberta.ca
}

\begin{abstract}
The discussion presents a framework of concepts that is intended to account for the rationality of semantic change and variation, suggesting that each scientific concept consists of three components of content: 1) reference, 2) inferential role, and 3) the epistemic goal pursued with the concept's use. I argue that in the course of history a concept can change in any of these components, and that change in the concept's inferential role and reference can be accounted for as being rational relative to the third component, the concept's epistemic goal. This framework is illustrated and defended by application to the history of the gene concept. It is explained how the molecular gene concept grew rationally out of the classical gene concept despite a change in reference, and why the use and reference of the contemporary molecular gene concept may legitimately vary from context to context.
\end{abstract}

This essay presents a framework of concepts that is designed to account for the rationality of semantic change. Though many previous discussions of conceptual change have emphasized the notion of reference, I argue that an adequate account has to acknowledge several semantic properties. More precisely, I suggest that each theoretical concept consists of three 
components of content: 1) the concept's reference, 2) its inferential role, and 3) the epistemic goal pursued by the concept's use. While reference and inferential role have been recognized by previous theories of concepts, I introduce the epistemic goal pursued by a term's use as a genuine semantic property of a term as it accounts for the rationality of semantic change and variation, in particular change in a term's inferential role and reference.

After the presentation of this framework and how it is intended to account for semantic change in Section 1, the remainder of the paper defends and illustrates the framework by application to a concrete case from biology: the change of the gene concept. The first part of this case study (Section 2) discusses how the molecular gene concept grew out of the classical gene concept. While the transition from classical to molecular genetics has been subject to extensive discussion, previous accounts have been less explicit about the rationality of the semantic change that occurred with the advent of the molecular gene concept. I explain why the transition from the classical to the molecular gene concept was rational despite a change in the reference of the term 'gene'. The second part of the case study (Section 3) addresses the development of the molecular gene concept in the last two decades, which has only recently attracted philosophical debate. A result of the many challenging empirical findings in molecular genetics and recently in genomics is that nowadays the term 'gene' is used in a varying fashion by molecular biologists. Different characterizations of what a gene is are used by different biologists and the contemporary molecular gene concept may vary in reference from context to context. I show why the term's context-sensitive usage and reference promotes scientific practice.

\section{A framework of concepts to account for the rationality of semantic change}

Traditional accounts of conceptual change in science have been developed as replies to the incommensurability challenge stemming from the work of Kuhn and Feyerabend. Kuhn 
(1962) argued that substantially different theoretical approaches ('paradigms') may use a given theoretical term with radically different, incommensurable meanings, so that communication across two paradigms and rational choice of one such theory over the other is impossible. Feyerabend $(1962,1970)$ claimed that the content of two theories containing incommensurable concepts cannot be compared. The standard reply to the incommensurability challenge has been to focus on the reference of scientific terms (Scheffler 1967; Putnam 1973; Fine 1975; Devitt 1979). Even if a term is used with different meanings in two theories, the term may nonetheless refer to the same entity across these theories, so that the theories can potentially make conflicting claims about this common referent and are thus comparable. Originally a purely causal theory of reference was favored, as it provides an explanation of how different scientists can refer to the same entity despite fundamentally different beliefs about this phenomenon (Sankey 1994; Boyd 2008).

However, despite its philosophical importance, the notion of reference alone cannot account for all issues surrounding semantic change. Even if a term's reference is stable across history, its definition may be subject to change. Section 2 will discuss the way in which the molecular gene concept grew out of the classical gene concept; and each of these concepts offers a quite different characterization of what a gene is. The philosopher has to account for how scientists can legitimately modify a term's definition. Likewise, a term’s usage may vary across different disciplines. Nowadays the molecular gene concept is used differently by different molecular biologists, and different characterizations of genes are preferred (Section 3). The philosophical task is to understand why such a variation in a term's meaning need not lead to a breakdown of communication across different researchers. Yet an account of reference alone (even if it ensures stability of reference) does not solve Kuhn's original challenge- how communication across different approaches and rational theory choice is possible despite variation in meaning — and it does not explain how it can be rational for scientists to modify the definitions of terms. Furthermore, even the very reference of a term may be subject to rational change. For instance, while the extensions of the classical and the 
molecular gene concept overlap, these concepts differ in reference as there are classical genes that are not molecular genes and vice versa (Section 2). In contemporary molecular biology, the reference of the term 'gene' may even shift from context to context—a phenomenon to be described and explored in Section 3. Using the notion of reference alone, it is impossible to explain how change in a term's reference can be rational, and why context-sensitive reference need not prevent communication and can be beneficial to scientific practice.

To be sure, some accounts of conceptual change have gone beyond the mere study of reference. The most sophisticated such theory is arguably Philip Kitcher’s. Kitcher (1978, 1982) introduced the notion of a 'mode of reference' as the way in which the reference of a term token is determined. The central idea is that for different tokenings of a term reference may be fixed in different ways (even if the referent is the same for these tokenings). Reference may be fixed causally (by causal interaction with the referent), but also descriptively (by using a description to pick out the referent), or by inheriting reference from past usage. There can be distinct coreferential modes of reference, e.g., different descriptions picking out the same entity, or an entity being picked out causally and descriptively. Kitcher suggested that concepts are reference potentials, where the reference potential of a term type is the set of modes of reference associated with this term. Assuming that the reference potential of a scientific term typically contains many modes of reference at any point in time, on this approach conceptual change is the acquisition of new modes of reference (their addition to the reference potential), or the abandonment of inadequate modes of reference (their deletion from the reference potential). Studying the change in a term's reference potential provides a much more fine-grained study of conceptual change than a mere consideration of the term's reference. Kitcher (1982) applied this framework to the history of the gene concept from classical to early molecular genetics. However, while he gives an account of which novel modes of reference came to be tied to the gene concept at certain points in time (how the meaning of 'gene' changed), his philosophical framework does not explain why the adoption 
of distinct modes of reference was rational (why change in the term's definition/meaning and possibly reference was warranted).

A term (a syntactic entity) as used in a certain context has semantic properties or content- the associated contentful mental representation is called a 'concept'. A change in the concept is a change in the term's semantic properties / the mental representation's content. The aim of the present paper is to work towards a framework of concepts that accounts for the rationality of semantic change and variation. To this end, I suggest that each theoretical term possesses three semantic properties, i.e., each theoretical concept consists of three components of content: 1) the term's reference, 2) its inferential role (analogous to Kitcher's reference potential), and 3) the epistemic goal pursued with the term's use. Different semantic properties are to be acknowledged because in the course of history a term may change in any of these properties (and it can possibly change in one without change in the others), and because different semantic properties serve different philosophical functions. In particular, the notion of the epistemic goal pursued by a concept's use is introduced here and considered a component of a concept as it is vital to account for the rationality of semantic change, more precisely, change in a term's inferential role and reference. Thereby change in one of a term's semantic properties can be counted as rational relative to another semantic property.

Inferential role is my notion for what has been called a term's meaning (sense, intension), and fairly similar to what Kitcher dubs a term’s reference potential. To a first approximation, it consists in a subset of the beliefs scientists have about the term's referent (those beliefs that are meaning-constitutive, and that determine the term's reference). I take over the label 'inferential role’ from inferential role semantics (also called conceptual role semantics), which—as one traditional semantic account—defines a concept's content in terms of how it figures in inference, or more broadly how it figures in reasoning, perception, and action (Block 1998; Field 1977; Harman 1987). Different attempts to draw a line between meaningconstitutive and other inferences, such as an analytic-synthetic distinction, have been subject to criticism. Rather than offering a theoretical account on this long-standing semantic 
question, I start with the assumption that the rationale for ascribing a 'sense' (inferential role) to a term is to understand the linguistic behavior of agents, which yields the following practical guideline. On my particular approach, a term's inferential role consists in those inferences and explanations in which the term occurs (as endorsed by the users of the term at a given time) and that account for the term's successful use in scientific practice. A concept supports scientific inference, explanation, and discovery; and inferential role is that component of a concept's content (reflecting empirical knowledge) that explains how the concept supports these features of scientific theorizing and practice. In the sections to follow, I will lay out what inferential roles were tied to the term 'gene' at different points in time, defending my account in this concrete case. Here I note that a term's inferential role, as I construe it, is more inclusive than a theoretical 'definition' which scientists may put forward. For in addition to a simple theoretical characterization, there are further features relevant for a term's successful usage. This may include central criteria that scientists need to find and investigate the term's referent. As a result, a historical change in a term's definition entails a change in its inferential role (but not necessarily the other way round), so that also on my account any change in a term's definition is a semantic change to be accounted for.

I do not assume that every person possessing a certain concept endorses the very same set of inferences and explanations that are part of the concept's inferential role. For different scientists may make use of different, yet complementary criteria. Due to the division of labor, it may in fact be significant for a concept's successful use in a scientific field that different scientists possess different epistemic abilities and exhibit them making different inferences, so that the concept's inferential role is heterogeneous and spread out over the whole community. While in his early work Kuhn assumed that differences in meaning are intrinsically problematic, some instances of semantic variation may be beneficial to scientific practice. (Later I will explain how my notion of 'epistemic goal' contributes to understanding this.) As a result, a concept and its inferential role need not be what an individual has grasped-a putative analytic statement shared by all users of the concept—but a concept's inferential role 
is determined by the linguistic and scientific practice of an overall community. In the course of history, a term's inferential role may change due to change in how the researchers making up this community use the term. As on traditional accounts, inferential role- together with the external world—determines reference. I view inferential role as a relevant component of a concept, as its philosophical function is to account for how a concept makes successful practice possible. (The property of reference as the mere fact that a term has a certain referent does not explain how this term underwrites successful practice. Rather, features determining reference such as inferential roles, which reflect empirical knowledge, do so.)

While previous theories have discussed reference and inferential role, most central to my philosophical discussion is the epistemic goal pursued by a concept's use, which I introduce the as a third component of conceptual content, as this feature accounts for the rationality of semantic change. It is well-known that a scientific community pursues various epistemic goals, and that an epistemic goal may be specific to a particular subdiscipline (e.g., the explanation of cell metabolism is a goal of biochemistry and to some extent of physiology, but not of evolutionary biology or ecology). While a scientific community often uses several concepts and theoretical resources to pursue a particular explanatory or investigative goal, in some cases such an epistemic goal (or a set of epistemic goals) can be tied to an individual scientific concept, in that the rationale for the introduction or continued use of a central theoretical concept is to pursue this epistemic goal. One concept may be used for scientific inferences of a certain kind (e.g., the homology concept of evolutionary biology is used for the establishment of phylogenetic trees), another concept may be used for a certain type of explanation (e.g., the concept of natural selection is needed to account for evolutionary adaptations). Still another concept may be used for experimental discovery of a certain kind (e.g., in molecular biology the notion of sequence similarity among genes, often called 'sequence homology', is used to arrive at estimates as to how novel systems can be most effectively investigated given knowledge of previously studied systems). My tenet is not that every scientific concept can be assigned a unique epistemic goal or a clearly delineated set of 
epistemic goals, or that this idea can be fruitfully applied to all scientific fields. Rather, my claim is that epistemic goals can be assigned to those central concepts (at least in biology) that underwent conceptual change, such that this semantic change can be explained in these terms. The fact that a certain scientific term is used for a particular goal need not be determined by how an individual scientist uses this term; rather, it is determined by the term's use by a scientific community. Within an overall field of science, there may be variation regarding the epistemic goal(s) pursued by a term's use, and thereby this component of a concept can also be subject to change in the course of history. As the structure of this variation may be an important determinant of semantic change, it ought to be taken into account by the philosopher, as my discussion on the contemporary molecular gene concept illustrates below.

When a term's definition changes, entailing change in the term's 'meaning' (inferential role on my account), it is not sufficient to explain this by pointing out that scientists have acquired novel, well-confirmed beliefs. For the acquisition of new beliefs about a term's referent—as it occurs every day—need not lead to a change in the very definition of the term. The rationality of semantic change (change in meaning) is to be accounted for over and above change in belief. The additional philosophical ingredient that does this is the notion of a concept's epistemic goal, in that the epistemic goal pursued by a term's use sets the standard for which changes in the term's inferential role and reference can count as epistemically warranted. While a concept's inferential role is the set of inferences and explanations currently supported by the concept, the concept's epistemic goal is the kinds of inferences and explanations that the concept is intended to support. A concept may be used to pursue certain theoretical, explanatory, or investigative goals, even though at the present time the conceptits inferential role reflecting current empirical beliefs_cannot adequately yield the intended epistemic product (inferences, explanations, discoveries), so that the concept's present inferential role does not fully meet its epistemic goal. Once significant novel empirical beliefs become available, the inferential role may change (possibly leading to a revision of the concept's definition), and this change in inferential role is rational provided that the revised 
inferential role (of the modified variant of the concept) meets the epistemic goal to a higher degree than the previous inferential role (of the original variant of the concept). Any epistemic goal in science comes—given empirical background knowledge-with implicit criteria specifying what it involves to adequately (or more adequately) satisfy the goal. For instance, if the goal is to explain a certain phenomenon, then the particular scientific context specifies what makes one explanatory attempt better then another one. (The degree to which one past scientific account actually met an epistemic goal pursued by scientists at this point in the past depends on factors besides what scientists believed back then, see pp. 22-23 below.)

In a nutshell, given a term's stable epistemic goal, a change in the term's inferential role (e.g. its definition) is warranted if due to the new inferential role the epistemic goal is met to a larger extent than hitherto. A change in a term's reference is rational if it occurs due to a change in the term's inferential role (which together with the world determines reference), provided that the change in inferential role is rational. To be sure, this basic scheme explains semantic change assuming a stable epistemic goal, yet change in a concept's inferential role or reference can be accompanied by a change in the concept's epistemic goal. The discussion in Section 2 explains based on a concrete case (the transition from the classical to the molecular gene concept) how to handle the more complicated situation of change in epistemic goal.

The rationale for the introduction of this third component of a concept can be made plain as follows. Many previous accounts of concepts have construed a concept in terms of certain beliefs about the referent—a definition, analytic statements, Kitcher's descriptive modes of reference-which my framework acknowledges as a concept's inferential role. Yet apart from asking what beliefs scientists have at a certain point in time, a further vital question is what they attempt to achieve by forming those beliefs, using a term in a certain way, and tentatively putting forward (and revising) its definition. For this latter feature, which I call the epistemic goal pursued by a term's use, provides a handle on understanding the rationality of semantic change. Dudley Shapere (1982) argued that in conceptual change, more important than a stability of meaning or reference is a continuity due to a chain of reasons. Nancy Nersessian 
(1984) elaborated this in the case of the concept of 'electromagnetic field', by breaking down this concept's meaning (inferential role in my terminology) into different parts (e.g., function, structure, causal power) and tracking the historical change of each such part, while viewing different historical stages of each part connected by 'chain-of-reasoning connections'. My account can be viewed as fleshing out what these 'reasons' justifying conceptual change are in some contexts, by making explicit that apart from a changing meaning (inferential role) there is an epistemic goal which is tied to a concept's use and which is the property setting the standards for which changes in meaning are rational. ${ }^{1}$

The basic framework of concepts sketched here will be illustrated and defended by application to a concrete case from biology — the change of the gene concept. In the following sections, I lay out which referent, inferential role, and epistemic goal were associated with the term 'gene' at certain points in history, and how each of these three components of content changed. While some previous discussions assumed that stable reference is a precondition for conceptual progress, I show that both change in a concept's inferential role and reference can be understood as rational. Thus, the aim of the case study is to show in a concrete situation how the three semantic properties (reference, inferential role, epistemic goal) can be ascribed to a scientific term, and how their change can be philosophically understood.

I conclude this section with a remark on concept individuation. If one assumes the existence of clear-cut conditions of concept individuation, a term as used by two different persons (e.g., scientists from different fields or different historical periods) either expresses the same concept or two distinct concepts. But on my account, the use of a scientific term may vary regarding three semantic properties (reference, inferential role, epistemic goal), so that any one of them, or any combination thereof could form the basis of concept individuation. As in different philosophical contexts different semantic properties may be more relevant, different individuation choices could in fact be legitimate. While accounts of how to individuate concepts (e.g. by means of an analytic-synthetic distinction) have proven to be unsatisfactory or incomplete, many philosophers still assume the existence of unique and non- 
arbitrary individuation conditions. Yet from my perspective, labeling two contexts in which a term is used as expressing the 'same concept' or two 'different concepts' is not of much philosophical relevance. Instead, what is important is to lay out how exactly two uses of a term differ regarding the various semantic properties. If there is a certain variation in inferential role across different contemporary scientists using the term, then the relevant question is to what extent communication across these different persons and term uses is possible, and in which contexts communication is hampered. If the term's inferential role or reference has changed in the course of history, one ought to explain why this semantic change was rational. An answer to this question has to be given and can be given independently of intuitions as to whether the semantic change consists in a previous concept giving rise to a distinct concept, or whether the semantic change occurs internal to an enduring concept. In the case study below I use such labels as the 'classical molecular gene concept' and the 'contemporary molecular gene concept' simply to distinguish the use of the term 'gene' in different points of time, or to make explicit to which scientific contexts (in which the term has been used) I refer. My task is to lay out how different uses of the term differ in various semantic properties, and to account for the rationality of this semantic change and variation.

\section{From the classical to the molecular gene concept}

As the transition from classical to molecular genetics has been subject to extensive philosophical study, my discussion focuses on those issues where it can add something to the existing literature. Previous accounts have discussed what different gene concepts have been

used and how such a concept defines what genes are, thereby covering what I call a concept's inferential role. In fact, Ken Waters's (1994) account of the classical and the molecular gene concept provides the basis for my construal of the inferential roles of these two concepts. I complement this by an account of the concepts' epistemic goals, so as to be able to account 
for the rationality of this instance of semantic change (involving a change in reference), an issue that previous discussions have addressed only implicitly.

The classical gene concept emerged at the beginning of the $20^{\text {th }}$ century. On my account, throughout the period of classical genetics the epistemic goal pursued by this concept's use was the prediction of patterns of inheritance. The concept's inferential role, in contrast, was subject to change reflecting improvement in the beliefs about the role of classical genes in inheritance. In the 1900s and 1910s, the gene concept (its inferential role) embodied empirical misconceptions, as a consistent distinction between genotypic units and phenotypic characters had yet to be established, and sometimes the erroneous assumption was (implicitly) made that the relation between genes and Mendelian characters was one-one (Carlson 1966; Weber 2005). Once the classical gene concept was fully established in the 1920s and 30s, its inferential role permitted a fairly adequate prediction of patterns of inheritance. This gene concept defines classical genes (alleles) functionally; the inferences that characterize the classical gene concept are those that specify how genes bring about patterns of inheritance. More precisely, the inferential role includes the genotype-phenotype distinction, the idea that genetic loci are arranged in linkage groups, beliefs about the basic principles specifying the transmission of genes (linkage, recombination, segregation), and ideas about the relation between genotype and phenotype (such as dominance and recessiveness). The classical gene concept did not embody beliefs about the material nature of genes, apart from the idea that genetic loci are associated with chromosomal locations (which permitted geneticists to account for double crossovers so as to establish linear genetic maps).

To be sure, classical geneticists did have beliefs about the material and internal structure of genes. Yet researchers widely disagreed about these matters (Carlson 1966), and most of these assumptions turned out to be wrong. Consequently, a construal of the classical gene concept's inferential role that does not include beliefs about the physical structure of genes focuses on those beliefs about genes on which different researchers agreed, pointing to a component of conceptual content that was shared among scientists. Moreover, the features 
that I include in the inferential role are those that are needed to account for how the classical gene concept was successfully used in biological practice (see page 5). For these ideas provided the epistemic access to genes, in that they permitted different geneticists-whatever their beliefs about the physical nature of genes — to detect and identify genes (based on inheritance patterns shown in mutation and breeding experiments), paving the way for further investigation. The inferential role also supported the prediction of patterns of inheritance, as demanded by the concept's epistemic goal: from the parents' genotype it is possible to infer the genotype distribution of the offspring generation, and from this the phenotype distribution of this filial generation.

It was completely unknown to classical geneticists how genes bring about their phenotypic effects. Furthermore, they knew that an allele may influence several Mendelian characters, and that each phenotypic character develops based on the action of many alleles (Morgan et al. 1915). It could be shown that a given classical gene is involved in the development of a certain character, but it was even unknown what the individual phenotypic contribution of this gene is that distinguishes its effect from the contribution of the other genes involved in the production of the character (Waters 1994). Thus, a mechanistic explanation of how an individual gene brings about its effect was impossible. Yet classical geneticists could explain phenotypic differences in terms of genetic differences. A single gene is a cause of a certain phenotypic feature as one cause among others, so that a genetic difference between two individuals that are otherwise genetically identical leads to a characteristic phenotypic difference (Morgan et al. 1915, 265-266; Sturtevant 1915).

The transition from the classical gene concept of the 1920s and 30s to the molecular gene concept of the 1960s and 70s was brought about by findings that shed light on the function and structure of individual classical genes. For instance, in the 1940s ingenious experiments on microorganisms offered evidence that mutations in certain individual genes led to missing or defective enzymes. Thereby it was shown for special organisms and genes what the gene's function and individual phenotypic contribution is. This led to the formulation of the 
one gene-one enzyme hypothesis, which assumed that the primary effect of each gene is the production of a particular enzyme (Beadle and Tatum 1941). Due to the discovery of the structure of DNA (Watson and Crick 1953), it became clear that genes are linear sequences of DNA nucleotides. Studies on viruses and bacteria permitted a more fine-grained analysis of mutation and recombination events, thereby shedding light on gene structure. It turned out that mutations can be due to changes in individual nucleotides as mere parts of genes, and that recombination occurs not only between but also within genes. Following the work of Benzer (1957), genes came to be viewed as cistrons, where a cistron is a continuous stretch of DNA that has an independent physiological effect, experimentally shown by the cis-trans test. All these findings led to a change in the inferential role of the term 'gene', including the one gene-one enzyme hypothesis coming to be refined to the one cistron-one polypeptide hypothesis.

Growing out of the classical gene concept during this transitional period, what is commonly called the molecular gene concept was established in the 1960s and 70s. Molecular genetics is not concerned with accounting for patterns of inheritance; instead the aim is to understand the operation of molecular, cellular, and developmental mechanisms, thereby addressing processes taking place within organisms, often within single cells - rather than the intergenerational processes of classical genetics. On my account, the epistemic goal pursued with the use of the molecular gene concept is to explain how genes bring about their molecular products. While the inferential role of the classical gene concept did not include substantial beliefs about the material nature of genes, a structural characterization of genes is vital for the molecular concept. For the structure of genes accounts for their causal capacities and molecular function (the latter being demanded by the epistemic goal). Molecular biology in general explains by means of the way in which various substances interact in mechanisms based on their structure-function relationships. I construe the inferential role of the molecular gene concept to involve the idea that a gene is a continuous sequence of DNA that is transcribed to a corresponding linear sequence of mRNA, which in turn is translated to a 
polypeptide, where the latter's amino acid sequence is determined by the nucleotide sequence of the mRNA in accordance with the genetic code. The way in which the linear DNA sequence determines (codes for) the sequence of its RNA or polypeptide product is an essential part of gene-based explanations in molecular biology (Waters 1994). In addition, the concept's inferential role includes knowledge about the mechanisms relevant for gene expression such as how transcription occur, for instance the idea that genes are preceded by promoter sequences which interact with transcription factors to initiate the expression of a specific gene in certain cells. In the 1970s, genes were often identified with open reading frames (ORFs), where an ORF is a continuous segment of DNA delimited by a start and stop codon and preceded by a promoter. While the relation between classical genes and gross phenotypic characters is many-many, a significant innovation of the molecular gene concept was to focus on a more proximal effect of genes (their molecular products), which-given the assumption of a one-one relation between molecular genes (structurally defined) and their products-aligned gene structure with gene function to a larger extent than previously.

As mentioned above, the classical gene concept could explain phenotypic differences between individuals (in terms of their genetic differences), but supported neither an explanation of the phenotypic contribution of individual genes nor a mechanistic explanation of how genes bring about their effects. The molecular gene concept fills this gap. As its inferential role includes a specification of the molecular structure of genes, the molecular concept supports a direct, causal-mechanistic explanation of how genes bring about their molecular products, and — together with other concepts—an explanation of development and the causal origination of phenotypic characters. As the molecular concept supports explanations that the classical gene concept did not support, we get a clear sense of conceptual progress occurring due to the semantic change (in particular change in inferential role) that the term 'gene' underwent in the transition from classical to molecular genetics.

However, while the molecular gene concept largely replaced the classical concept, it did not eliminate the latter. The classical gene concept is still prominently used in population 
genetics, and it is sometimes used in molecular genetics (Vance 1996; Moss 2003). Marcel Weber (2005) gives an insightful discussion of how molecular biologists—-while on the theoretical level favoring a molecular construal of genes — used on the experimental level both the classical and the molecular gene concept in tandem in the historically significant method of identifying and sequencing molecular genes for the first time. Reduction of a historically earlier theory to a later theory is a sufficient condition for conceptual progress, yet it is only one way in which progress can occur. The debate about reduction in genetics showed that classical genetics cannot be reduced—in the sense of theory reduction as endorsed by Schaffner (1969)—-to molecular biology (Hull 1974; Kitcher 1984; Brigandt and Love 2008). Thus, the transition from the classical to the molecular gene concept is an instance of progress that occurred without the molecular concept fully replacing or reducing the classical concept. Rather than attempting to show that reduction is possible after all, a more fruitful project is to study the interplay between both concepts in contemporary practice (as Weber does), and to understand how the molecular could grow out of the classical concept (Kitcher 1982).

Moreover, stability in a term's reference has often been viewed as a necessary condition for conceptual progress, as discussions surrounding issues such as realism, incommensurability, and the pessimistic meta-induction show. The motivation for this idea is that only in the case of stable reference can one say that later scientists have an improved conception of the very same entity that earlier scientists referred to, whereas in the case of reference change the assumption is that our novel conception is simply about another object. However, the reference of the gene concept has changed throughout its history (as acknowledged implicitly by Kitcher 1982 and explicitly by Burian et al. 1996). While the extensions of the classical and the molecular gene concept strongly overlap, Weber (2005) discusses in detail why there are classical genes that are not molecular genes and vice versa (since classical genes are defined in terms of their phenotypic function, while molecular genes are defined in terms of product-coding structures, both concepts can pick out different genetic segments at complex eukaryotic loci). Weber argues that geneticists have tracked not one but 
several (overlapping yet distinct) kinds by the term 'gene', and uses the notion of 'floating reference' for the idea that the reference of the gene concept changed constantly, though in a gradual fashion, suggesting that “this floating of the term's reference seems not to have diminished its theoretical importance or practical usefulness” (Weber 2005, 224). Thus, a significant task for any account of conceptual change in genetics is to explain how the molecular gene concept could rationally develop out of the classical gene concept despite a change in reference.

In the transition from the classical to the molecular gene concept, all three components of conceptual content changed: the reference of the term 'gene', its inferential role, and the epistemic goal pursued by the term's usage. I have introduced the notion of a concept's epistemic goal as an additional component of a concept since it is this component that sets the standards for which changes in inferential role (definition, meaning) and reference can count as rationally warranted. For instance, a concept’s epistemic goal may specify a certain explanandum, while the concept's current inferential role (reflecting present scientific knowledge) provides a partial explanans, which at this point does not fully explain the explanandum. The basic idea introduced in the previous section is that given a concept's stable epistemic goal, change in the concept's inferential role-and corresponding potential change in reference--is rational if the revised inferential role meets the epistemic goal to a higher degree than the previous inferential role. However, such a straightforward account based on a stable epistemic goal cannot quite apply to how the molecular gene concept developed out of the classical gene concept, as in this case also the epistemic goal shifted. Change in inferential role can nonetheless be rational in such a situation, provided that the modification in epistemic goal occurred in gradual fashion, and each stepwise change in inferential role counts as rational relative to the epistemic goal that obtained immediately prior to this change in inferential role. Consider a scientific term's use at time slices $t_{0}, t_{1}, \ldots t_{n}$, where the epistemic goal at $t_{0}$ and $t_{n}$ differ significantly with gradual change occurring in between. Then a change in the term's inferential role (and possibly reference) from $t_{0}$ to $t_{1}$ is 
rational if the revised inferential role at $t_{1}$ meets the epistemic goal at $t_{0}$ to a larger degree than the inferential role at $t_{0}$. Thus, at $t_{1}$ one evaluates relative to the previous epistemic goal at $t_{0}$ (which yields the same verdict as evaluating relative to the current epistemic goal at $t_{1}$, as the latter is similar to the epistemic goal at $\mathrm{t}_{0}$ ). In contrast, an evaluation relative to the epistemic goal at $t_{n}$ would beg the question since in the assessment of the transition to $t_{1}$ one had to appeal to an epistemic goal of concept use that did not obtain yet. Proceeding this way permits assessing the rationality of semantic change in a stepwise fashion from $t_{0}$ to $t_{n}$.

This scheme applies to the history of the gene concept as follows. The epistemic goal of the classical gene concept was the prediction of patterns of inheritance. In classical genetics, genes were often viewed as the units of mutation, recombination, and function. For all three processes are needed to obtain patterns of inheritance. One aspect of this is the idea that genes are units of function, i.e., material entities that can be inherited and make an independent physiological contribution. Research in the 1940s and 50s (the transitional period between classical and molecular genetics) provided insights into the fine-structure of genes—still understood as classical genes — and the causal contribution of individual genes, e.g., the findings that genes are DNA sequences and that mutation and recombination can occur within cistrons, and the hypothesis that each gene codes for an enzyme. This empirical knowledge became integrated into the gene concept, leading to a change in the term's inferential role and its definition (change in the term's 'meaning'). This first step of semantic change was rational as it was based on those items of empirical information about genes that were relevant relative to one aspect of the epistemic goal pursued with the classical gene concept, namely, understanding the physiological impact of genes (which were among other things conceived of as units of function).

This initial change in inferential role and the theoretical changes in genetics also led to a modification of the epistemic goal pursued. Geneticists came to focus exclusively on the physiological and later molecular function of genes, largely abandoning the aim of explaining patterns of inheritance. Findings of the 1960s and 70s about the structures underlying gene 
function (e.g., open reading frames, the genetic code, promoters and regulatory sequences) led to further change and eventually to the establishment of the molecular gene concept as a notion different from the classical concept. The epistemic goal pursued by the use of the molecular gene concept is to account for the molecular function of genes, which is a modification of what used to be only one among several aspects of the classical concept's epistemic goal. (This change in the term's epistemic goal was scientifically warranted as an adequate account of patterns of inheritance had already been provided, and addressing the molecular function of genes is necessary for explaining cellular, physiological, and developmental processes.) The overall semantic change endowed biologists with an increasing ability to explain genetically based phenomena. It was progressive as the concept's inferential role was integrating those discoveries about gene structure and function that permitted a better explanation of the phenomena demanded by the concept's (current) epistemic goal. A sideeffect of this rational and progressive change in the inferential role of the term 'gene' was that reference shifted. The molecular and the classical concept individuate genes in different ways, and as it turned out, classical and molecular genes are strongly overlapping, yet non-identical categories. Originally, this reference change occurred largely unbeknownst to geneticists.

\section{From the 'classical' molecular gene concept to the contemporary molecular gene concept}

The molecular gene concept of the 1970s is nowadays often called the 'classical' molecular concept, as semantic change has occurred (and is still taking place) due to novel empirical findings in genetics and genomics (Griffiths and Stotz 2007). While the classical molecular concept featured a unified vision of genes, the contemporary gene concept is a more fragmented concept, in that different scientists offer different characterizations of what genes are and use different structural criteria of individuating genes, leading to a situation where the term 'gene' refers to different categories in different contexts. After briefly laying out some 
findings that led to this instance of conceptual change, I explain why the recent change in the term's inferential role and reference was rational. In this section, however, my semantic account focuses on semantic variation. I suggest that the term's current context-sensitive usage and reference promotes biological practice, and that at the same time there is some unity behind these different uses_-in opposition to calls to abandon the term 'gene'.

The classical molecular gene concept featured the idea of a one-one relation between genes and gene products, moreover assuming that each gene is an open reading frame (ORF), so that genes form a unique structural kind. However, it turned out that in eukaryotes the relation between genetic elements and gene products is many-many, and that genes are rather a set of many overlapping, yet different structural categories, so that biologists may nowadays refer to one of these categories in one context, but another one in a different situation. The one-many relation between genetic elements and gene products results from alternative splicing. In the first step of gene expression, transcription, a continuous stretch of DNA (an ORF or so-called transcription unit) is transcribed to pre-mRNA (the primary transcript). In the process of splicing certain segments—called introns—are removed (spliced out), and the remaining segments_-called exons_-are joined, forming the mature mRNA. In the final step of gene expression, translation, the mature mRNA is translated to a polypeptide. Thus, a total gene is a DNA stretch consisting of several alternating coding exons and non-coding introns. This continuous sequence is transcribed to pre-mRNA, and then the introns are spliced out, yielding a mature mRNA as a sequence of exons that are translated. In alternative splicing, only some exons of a gene's total exon set are joined to form the mature mRNA that is translated; and which exon subset is used varies from splicing instance to splicing instance. In other words, different transcripts of a token gene (different pre-mRNAs with the same sequence) yield mature mRNAs consisting of different exon combinations (thus different in type). Consequently, one genetic element transcribed as a unit (hence one gene?) can yield many different products, violating the traditional assumption that each gene codes for exactly one product (Portin 1993; Leipzig et al. 2004). 
There is also a many-one relation between DNA elements and gene products. In transsplicing (also called intergenic splicing), the mature mRNA is produced from several premRNAs, where parts of two or more pre-mRNAs are fused together by splicing to form a mature mRNA, which is in turn translated to a polypeptide. In many cases this happens when each of two or more distinct ORFs (genes?), possibly located on different chromosomes, produces a pre-mRNA, and some parts of these pre-mRNAs differing in sequence are transspliced together (Finta and Zaphiropoulos 2000; Finta et al. 2002; Magrangeas et al. 1998; Zhang et al. 2003). In sum, in transcription an ORF always produces the same pre-mRNA, so that a one-one relation between genetic elements and primary transcripts holds. In the subsequent step of splicing, the causal sequence may branch (alternative splicing leading to several mature mRNAs) or merge (trans-splicing of several pre-mRNAs to one mature mRNA), so that there is a many-many relation between primary transcripts and mature mRNAs. Each mature mRNA is usually translated to a unique polypeptide (protein product). Overall, a many-many relation between genetic elements and their protein products obtains.

There are many more complexities regarding how various DNA elements code for a product, such as RNA editing and translational recoding (Gray 2003; Baranov et al. 2003), overlapping genes (Mottus et al. 1997), transcription in different reading frames (Sharpless and DePinho 1999), and antisense transcription (Dorn and Krauss 2003; Yelin et al. 2003). But this paper is not the place to discuss all challenges for traditional views of molecular genes influencing biologists' use of the term 'gene' (for philosophical reviews see Griffiths and Stotz 2007; Stotz 2006a, b; Stotz and Griffiths 2004). I focus on the many-many relation between DNA elements and gene products as the main ground of why biologists may individuate genes differently. In the case of alternative splicing, one may consider an ORF as one gene coding for different products, or — in particular if the products are biochemically very different—as many genes (one for each kind of product) that happen to be physically identical. As in the case of trans-splicing several distinct and non-contiguous genetic elements are involved in the production of a polypeptide, it is unclear whether several genes count as 
working together to bring about a product or whether the polypeptide is coded for by a single gene that happens to consist of several distinct units, possibly located at different regions of the genome. As a result, different biologists may consider a particular mereological part of the genome (possibly involving several non-contiguous segments) as constituting a single gene, as merely being a proper part of a gene, or as forming a collection of several genes.

Typically, such a choice as to how to individuate genes depends on the research context, i.e., the considerations about the structure or function of genes that govern a particular usage of the term 'gene'. For instance, as there is still a one-one relationship between DNA segments and primary transcripts (pre-mRNAs), yet a many-many relation between DNA segments and polypeptide products, researchers focusing on RNA as the product of interest will often individuate differently from biologists studying the polypeptide product. Some geneticists assume that DNA elements with distinct promoters have to count as distinct genes; others do not make this requirement. Some permit that a gene may have different products, yet count genetic elements that are trans-spliced together as distinct genes. Some view DNA elements that are trans-spliced together as one gene, unless both sense and antisense elements are joined in trans-splicing. Other relevant considerations are whether all separable genetic elements are translated, whether a genetic element that forms a product in conjunction with other DNA elements (trans-splicing) also produces another product on its own in other cellular contexts, how far apart the different DNA segments involved are, and how chemically diverse the different products are. Several such considerations can be combined to various specific characterizations of what a gene is. Each such way of individuating genes picks out a different category (though different such categories overlap extensionally), so that genes are not a unique kind, but a set of overlapping, yet different categories. One use of 'gene' refers to one such category, another use-made by a different scientist or the same person in a different research context—-may refer to another such category, leading to the reference of the term 'gene’ sometimes varying from context to context. 
Different philosophical accounts of how many distinct gene concepts are nowadays used in molecular biology or how to characterize them have been put forward (Burian 2004; Burian et al. 1996; Falk 1986, 2000, 2004; Keller 2000; Rheinberger 2000). Moss (2003) distinguishes two gene concepts, Griffiths and Stotz (2007) argue for there being three. While Waters $(2000,2004)$ argues that there still is a unified gene concept used in molecular biology, there are both a few biologists and philosophers suggesting that the gene concept is being abandoned or can be or should be eliminated (Gelbart 1998; Gray 1992; Kitcher 1992; Neumann-Held 1999). It is in fact the case that nowadays further terms apart from 'gene' are used to refer to certain genetic elements coding for RNAs and polypeptides (e.g., 'open reading frame', 'exon', 'transcription unit'), and the joint usage of different terms provides a less ambiguous way of specifying which aspects of gene structure and function are talked about on a particular occasion. At the same time, the term 'gene' is still widely used, and currently scientists react to novel and challenging discoveries by trying to absorb them into the gene concept rather then simply abandoning it (Fogle 2000). Instead of attempting to decide how many 'different' gene concepts there are, my semantic account contributes to the current literature by working toward two aims—-which are prima facie in tension: 1) to account for why the term 'gene' is legitimately used differently in different contexts and how this context-sensitive usage supports successful biological practice, and 2) to specify what different usages of the term 'gene' have in common, so as to account for why the term 'gene' is used across contexts and communication across contexts and biologists is still possible.

My account essentially invokes as a component of conceptual content the epistemic goal pursued by the molecular gene concept's use. In the case of the 'classical' molecular gene concept (of the 1970s), Section 2 argued that it is used to explain how genes bring about their molecular products. The same actually holds of the contemporary molecular gene concept. Thus, while in the last three decades semantic change occurred regarding the inferential role of the molecular gene concept (structural characterizations of genes and definitions of the term 'gene' have changed), there is an important element of conceptual stability: no change 
occurred as regards what biologists attempt to achieve when using the term 'gene', the epistemic goal is still to explain how DNA elements code for different molecular substances (RNAs and polypeptides). While this sameness of epistemic goal unites the molecular gene concept of the 1970s and the contemporary one, the change in the inferential role (and reference) that the term 'gene' underwent in the last decades has to be accounted for.

It is not sufficient to point out that geneticists have acquired novel and well-confirmed beliefs about genes and that this is why nowadays they legitimately make different statements involving the term 'gene'. The task is to account for why even definitions of this term have changed, the rationality of semantic change (change in inferential role) is to be accounted for over and above change in belief. This can be achieved due to the stability of the epistemic goal pursued by the molecular gene concept's use. In the 1970s, the assumption was that each open reading frame is expressed so as to result in exactly one gene product and that every product is coded for by a single ORF, leading to the standard definition of genes as ORFs. This was a structural definition of genes which together with knowledge about the mechanism of transcription and translation seemed to fully account for gene function-the concept's epistemic goal. Subsequent findings in molecular genetics and recently in genomics revealed that the structural basis of gene function is much more complicated in that the DNA elements coding for products form a structurally heterogeneous class. For example, a proper part of an ORF may code for a functional product, or several non-contiguous ORFs may be responsible for a single product. In concrete cases, various structural features bear on whether genetic elements are expressed and which set of genetic elements codes for a given product. Consequently, it was rational for geneticists to largely abandon the traditional definition of genes as ORFs and offer modified and more varied structural definitions for the term 'gene', as the modern characterization of gene structure provides an improved account of how genetic elements produce their products-thereby meeting the molecular gene concept's stable epistemic goal to a higher degree. Among the various new findings about genes (belief change), those that prompted and justified a redefinition of 'gene' (meaning change) were 
those pertaining to the structural basis of gene function. In the last decades the reference of the term 'gene' also changed somewhat (nowadays it refers not only to individual ORFs), and this change in reference is a by-product of the rational change in inferential role.

Not only has the unified definition of the classical molecular gene concept ceded to a more sophisticated account, but as nowadays different criteria as to how to individuate genes are used, the inferential role and reference of the term 'gene' can change from context to context. The notion of a concept's epistemic goal points to some conceptual unity: underlying the various different uses of this term is a common motivation-to account for gene function. Different structural characterizations of genes may pick out not quite identical categories, yet different biologists still count as addressing the same phenomenon, by being part of a common explanatory practice that attempts to gain an even better understanding of the genetic elements coding for functional products. The shared epistemic goal explains why different geneticists tend to react similarly to findings challenging definitions of genes, enabling some communication. Despite its heterogeneity, there is still some gene concept—or at least some important component of content—shared among molecular biologists. ${ }^{2}$

The notion of a concept's epistemic goal also provides the basis for explaining the variation in usage across contexts. Different biologists may spell out the shared generic epistemic goal in different precise ways. For instance, in one research context the focus may be on certain structural features of genes, such as sequences that usually lead to the initiation of transcription. In another context, scientists (e.g. RNA researchers) may be interested in the RNA transcript, while still others (e.g. protein biochemists) may focus on the polypeptide finally produced from a gene. It has become clear that RNAs are not only intermediates but like enzyme-forming polypeptides fulfill important cellular functions (Mattick 2003). But only the relation between DNA elements and polypeptides is many-many, often yielding different gene individuations depending on whether protein or RNA is the product of interest. As there is no single structurally delineated category that would explain the production of RNAs and polypeptides in all cases and a many-many relation between genetic elements and gene 
products holds, different scientific interests may pick out differently characterized entities. A context-sensitive usage of the molecular gene concept promotes biological practice, because there are different legitimate explanatory and investigative interests—specific epistemic goals - that may underlie the study of one and the same genetic region (and expression events involving this region), and each particular use has to rely on those criteria of gene individuation that are relevant for this genetic region and the explanatory interests. Given such a specific epistemic goal, using a broad consensus definition that includes only those features common to all genes (or the classical molecular concept) is often inadequate to determine how to delineate various DNA elements as genes in a particular genetic region. As a result, the reference of the term 'gene' can legitimately vary across uses. Moreover, the overall flexibility in term usage permits scientists to react to novel findings, thereby taking into account that science is addressing entities (e.g. genes) and phenomena (gene function) that still are not fully understood.

In spite of the strongly varying usage, communication across contexts is still possible-at least to a relevant extent. For biologists are aware of the flexible overall use of the gene concept, and the particular research context disambiguates in that within a certain context it is relatively clear what characterization and understanding of 'gene' is in play and which entity is actually referred to. If some biologist happens to be unaware of any feature of the epistemic context and biological case that underlies a statement or sentence involving the term 'gene' uttered or written by another geneticist, a precise understanding of what is meant is not possible. Yet even in such an extreme case a rough and generic understanding is available after all, as the different categories that the term 'gene' may refer to overlap extensionally, and different uses of this term have some basic features in common, including stereotypical properties of genes such as the idea that a gene is one or several DNA segments consisting of exons, being transcribed and often translated so as to code for a linear product, etc. Communication is never an all-or-nothing affair; and for understanding many statements involving 'gene' a general conception of genes is sufficient, whereas the understanding of 
some other statements makes it necessary to grasp the particular individuation criteria of genes used, so that some knowledge of the epistemic context and biological case is required. ${ }^{3}$

Apart from the general context that disambiguates the particular meaning in play, in their statements biologists can make use of other terms in addition to 'gene' (such as 'exon' or 'transcription unit') to offer a more explicit and precise characterization of which type of entity is meant. The somewhat monolithic classical molecular gene concept has ceded to a contemporary use of 'gene' that is one among several terms used to effectively talk about genes and their function. Given this, one may wonder why should one talk about 'genes' at all rather than eliminating this word in favor of a set of terms whose joint employment could support a less ambiguous use? My reply is that communication across various contexts is scientifically necessary, and scientific notions and claims ought to have a basic generality across contexts. This requires a shared terminology and understanding such as the current gene concept. In contrast, using solely terms that may promise precision but apply to a particular experimental context only would due to this specificity make communication across contexts difficult (Keller 2000). My claim that the flexible use of the gene concept facilitates current biological practice does not imply that the gene concept should be with us until the end of time. Future science may find terminologically and conceptually more appropriate ways to explain molecular function; but currently, often making use of a single term 'gene' in various situations, while at the same time using it differently, is an appropriate way to address a trade-off that exists between scientific precision and generality (communication) across contexts.

\section{Conclusion}

I suggested that each theoretical concept, at least each central biological concept, consists of three components of content: 1) the concept's reference, 2) its inferential role, and 3) the epistemic goal pursued with the concept's use. Different such semantic properties of a term 
are to be acknowledged because a term may change in any of them in the course of history (one property possibly changing without the others), and because different semantic properties fulfill different philosophical functions. For instance, the property of inferential role accounts for how a concept supports successful scientific practice and communication.

While previous semantic accounts have recognized reference and inferential role ('sense'), the novel feature of my approach is to introduce the epistemic goal pursued by a term's use as an additional semantic property of a term (as a component of a concept), because it accounts for the rationality of semantic change and variation. While a scientific community uses various conceptual resources to pursue its epistemic goals, in some cases such an epistemic goal (or a set of goals) can be tied to an individual concept as the rationale of its introduction. The epistemic goal of a concept consists in those theoretical, explanatory, or investigative aims that are pursued by this concept's use. Given a concept's stable epistemic goal, a change in its inferential role (e.g. a change in a term's definition) is rational provided that the revised inferential role (the inferences and explanations supported by the revised concept) meets the epistemic goal (the inferences and explanations aimed at) to a higher degree than the previous inferential role. A change in reference is likewise epistemically warranted if it results from a rational change in inferential role.

I defended and illustrated the framework of concepts by applying it to the gene concept. In the case of the transition from classical genetics to molecular genetics, all three components of content associated with the term 'gene' changed. Some previous discussions of conceptual change have assumed that conceptual progress presupposes that a term's reference is unchanging (while the beliefs about this referent improve). However, the transition from the classical to the molecular gene concept is an instance of conceptual progress involving a change in the reference of 'gene'. I suggested that the rationality of this change in inferential role (sense) and reference can be accounted for relative to the gradually changing epistemic goal pursued by the use of the term 'gene', where in a stepwise fashion the change in 
inferential role occurring in a shorter period of time is evaluated as rational with respect to the immediately preceding epistemic goal.

In the last three decades, semantic change has occurred in molecular biology-a change in the inferential role and reference of the term 'gene'. I accounted for this using the term's stable epistemic goal (explaining how genes generate their molecular products). Due to revised beliefs about the structural basis of gene function, the molecular gene concept's inferential role of the 1970s ceded to a more complex account of the structure of DNA elements. The latter provides a better explanation of how genes bring about their products, so that the modified inferential role meets the concept's epistemic goal to a higher degree, constituting rational semantic change.

Nowadays different biologists may prefer different accounts of what individuates genes, so that both the inferential role and the reference of the contemporary molecular gene concept can vary from researcher to researcher. The notion of the epistemic goal of a concept also accounts for semantic variation. I argued that the context-sensitive use and reference of the contemporary gene concept promotes biological practice, and explained this by the fact that the concept's generic epistemic goal shared across uses (providing some unity across variation) can be spelled out differently in different research contexts, where one such specific epistemic goal addresses a particular aspect of gene function, which calls for a contextdependent structural characterization of genes. I explained why using the term in such a varying fashion need not make communication impossible, and why there are still reasons for using 'gene' rather than eliminating it in favor of other terms.

Independently of whether the particular semantic framework proposed here offers a satisfactory account of the rich history and present situation of the gene concept, my discussion showed that any theory of conceptual change should be able to account for the rationality of change in a term's reference across history, and for a term's context-sensitive use and reference within a scientific community. 


\section{Notes}

${ }^{1}$ Formal epistemologists have recently argued that accounts of belief revision have to take into account an agent's research agenda (sets of open questions) apart from the agent's beliefs and the beliefs' entrenchment ordering (Olsson and Westlund 2006). The notion of research agenda bears similarities to the idea of epistemic goal; though while the former is used to account for (epistemic) change of belief sets, I invoke the latter to deal with (semantic) change of individual concepts.

${ }^{2}$ Philosophers of language may keep semantic content (sentence meaning) as what is shared across different usages apart from speech act content (speaker's meaning) as what is expressed in a specific context and may vary across uses (Cappelen and Lepore 2005). My account conforms to this distinction, yet goes beyond standard semantic accounts by assuming that a term's shared semantic content does not just consist in a meaning (inferential role) and a reference, but that there is also the epistemic goal as a shared feature of semantic importance.

${ }^{3}$ In the case of the molecular gene concept, the variation in the concept's specific epistemic goal across researchers is unified by a shared generic epistemic goal. Yet there may well be cases where different scientific disciplines use one and the same term to pursue quite different epistemic goals, where it is unclear how they are related. In this case, across these disciplines misunderstandings are likely to arise, as researchers either do not even realize that others use the term for quite different scientific purposes, or simply insist that the purpose for which they happen to use the concept is the real one. Such an instance of partial communication failure ought to be philosophically understood, and the notion of epistemic goal is germane to this as well. 


\section{References}

Baranov, P. V., Gurvich, O. L., Hammer, A. W., Gesteland, R. F. \& Atkins, J. F. (2003). Recode 2003. Nucleic Acids Research, 31, 87-89

Beadle, G. W. \& Tatum, E. (1941). Genetic control of biological reactions in Neurospora. Proceedings of the National Academy of Sciences USA, 27, 499-506

Benzer, S. (1957). The elementary units of heredity. In W. D. McElroy \& B. Glass (Eds.), A symposium on the chemical basis of heredity (pp. 70-133). Baltimore: John Hopkins Press

Block, N. (1998). Semantics, conceptual role. In E. Craig (Ed.), Routledge Encyclopedia of Philosophy, vol. 8 (pp. 652-657). London: Routledge

Boyd, R. (2008). Scientific realism. In E. N. Zalta (Ed.), The Stanford Encyclopedia of Philosophy (Fall 2008 edition). http://plato.stanford.edu/archives/fall2008/entries/scientific-realism/

Brigandt, I. \& Love, A. C. (2008). Reductionism in biology. In E. N. Zalta (Ed.), The Stanford Encyclopedia of Philosophy (Fall 2008 edition). http://plato.stanford.edu/archives/fall2008/entries/reduction-biology/

Burian, R. M. (2004). Molecular epigenesis, molecular pleiotropy, and molecular gene definitions. History and Philosophy of the Life Sciences, 26, 59-80

Burian, R. M., Richardson, R. C. \& van der Steen, W. J. (1996). Against generality: Meaning in genetics and philosophy. Studies in History and Philosophy of Science, 27, 1-29

Cappelen, H. \& Lepore, E. (2005). Insensitive semantics: A defense of semantic minimalism and speech act pluralism. Malden: Blackwell

Carlson, E. A. (1966). The gene: A critical history. Philadelphia: W. B. Saunders

Devitt, M. (1979). Against incommensurability. Australasian Journal of Philosophy, 57, 2950

Dorn, R. \& Krauss, V. (2003). The modifier of mdg4 locus in Drosophila: Functional complexity is resolved by trans splicing. Genetica, 117, 165-177

Falk, R. (1986). What is a gene? Studies in the History and Philosophy of Science, 17, 133173

Falk, R. (2000). The gene: A concept in tension. In P. J. Beurton, R. Falk \& H.-J. Rheinberger (Eds.), The concept of the gene in development and evolution (pp. 317-348). Cambridge: Cambridge University Press

Falk, R. (2004). Long live the genome! So should the gene. History and Philosophy of the Life Sciences, 26, 105-121 
Feyerabend, P. (1962). Explanation, reduction, and empiricism. In H. Feigl \& G. Maxwell (Eds.), Scientific explanation, space, and time (Minnesota Studies in the Philosophy of Science, vol. 3) (pp. 28-97). Minneapolis: University of Minnesota Press

Feyerabend, P. (1970). Against method. In M. Radner \& S. Winokur (Eds.), Analyses of theories and methods of physics and psychology (Minnesota Studies in the Philosophy of Science, vol. 4) (pp. 17-130). Minneapolis: University of Minnesota Press

Field, H. (1977). Logic, meaning, and conceptual role. Journal of Philosophy, 74, 379-408

Fine, A. (1975). How to compare theories: Reference and change. Noûs, 9, 17-32

Finta, C., Warner, S. C. \& Zaphiropoulos, P. G. (2002). Intergenic mRNAs: Minor gene products or tools of diversity? Histology and Histopathology, 17, 677-682

Finta, C. \& Zaphiropoulos, P. G. (2000). The human cyp2c locus: A prototype for intergenic and exon repetition splicing events. Genomics, 63, 433-438

Fogle, T. (2000). The dissolution of protein coding genes in molecular biology. In P. J. Beurton, R. Falk \& H.-J. Rheinberger (Eds.), The concept of the gene in development and evolution (pp. 3-25). Cambridge: Cambridge University Press

Gelbart, W. M. (1998). Databases in genomic research. Science, 282, 659-661

Gray, M. W. (2003). Diversity and evolution of mitochondrial RNA editing systems. IUBMB Life, 55, 227-233

Gray, R. D. (1992). Death of the gene: Developmental systems strike back. In P. E. Griffiths (Ed.), Trees of life: Essays in the philosophy of biology (pp. 165-210). Dordrecht: Kluwer

Griffiths, P. E. \& Stotz, K. (2007). Gene. In D. L. Hull \& M. Ruse (Eds.), The Cambridge Companion to the Philosophy of Biology (pp. 85-102). Cambridge: Cambridge University Press

Harman, G. (1987). (Non-solipsistic) conceptual role semantics. In E. Lepore (Ed.), New directions in semantics (pp. 55-81). London: Academic Press

Hull, D. (1974). Philosophy of biological science. Englewood Cliffs: Prentice-Hall

Keller, E. F. (2000). The century of the gene. Cambridge, MA: MIT Press

Kitcher, P. (1978). Theories, theorists, and theoretical change. The Philosophical Review, 87, 519-547

Kitcher, P. (1982). Genes. British Journal for the Philosophy of Science, 33, 337-359

Kitcher, P. (1984). 1953 and all that: A tale of two sciences. The Philosophical Review, 93, 335-373

Kitcher, P. (1992). Gene: Current usages. In E. F. Keller \& E. A. Lloyd (Eds.), Keywords in evolutionary biology (pp. 128-131). Cambridge, MA: Harvard University Press 
Kuhn, T. S. (1962). The structure of scientific revolutions. Chicago: University of Chicago Press

Leipzig, J., Pevzner, P. \& Heber, S. (2004). The Alternative Splicing Gallery (ASG): Bridging the gap between genome and transcriptome. Nucleic Acids Research, 13, 3977-3983

Magrangeas, F., Pitiot, G., Dubois, S., Bragado-Nilsson, E., Cherel, M., Jobert, S., Lebeau, B., Boisteau, O., Lethe, B., Mallet, J., Jacques, Y. \& Minvielle, S. (1998). Cotranscription and intergenic splicing of human galactose-1-phosphate uridylyltransferase and interleukin-11 receptor alpha-chain genes generate a fusion mRNA in normal cells: Implication for the production of multidomain proteins during evolution. Journal of Biological Chemistry, 273, 16005-16010

Mattick, J. S. (2003). Challenging the dogma: The hidden layer of non-protein-coding RNAs in complex organisms. BioEssays, 25, 930-939

Morgan, T. H., Sturtevant, A. H., Muller, H. J. \& Bridges, C. B. (1915). The mechanism of Mendelian heredity. New York: Henry Holt

Moss, L. (2003). What genes can't do. Cambridge, MA: MIT Press

Mottus, R. C., Whitehead, I. P., O'Grady, M., Sobel, R. E., Burr, R. H. L., Spiegelman, G. B. \& Grigliatti, T. A. (1997). Unique gene organization: Alternative splicing in Drosophila produces two structurally unrelated proteins. Gene, 198, 229-236

Nersessian, N. J. (1984). Faraday to Einstein: Constructing meaning in scientific theories. Dordrecht: Kluwer

Neumann-Held, E. M. (1999). The gene is dead - long live the gene: Conceptualising the gene the constructionist way. In P. Koslowski (Ed.), Sociobiology and bioeconomics: The theory of evolution in biological and economic theory (pp. 105-137). Berlin: Springer

Olsson, E. J. \& Westlund, D. (2006). On the role of the research agenda in epistemic change. Erkenntnis, 65, 165-183

Portin, P. (1993). The concept of the gene: Short history and present status. The Quarterly Review of Biology, 68, 173-223

Putnam, H. (1973). Explanation and reference. In G. Pearce \& P. Maynard (Eds.), Conceptual change (pp. 199-221). Dordrecht: Reidel

Rheinberger, H.-J. (2000). Gene concepts: Fragments from the perspective of molecular biology. In P. J. Beurton, R. Falk \& H.-J. Rheinberger (Eds.), The concept of the gene in development and evolution (pp. 219-239). Cambridge: Cambridge University Press

Sankey, H. (1994). The incommensurability thesis. Brookfield: Avebury

Schaffner, K. F. (1969). The Watson-Crick model and reductionism. British Journal for the Philosophy of Science, 20, 325-348 
Scheffler, I. (1967). Science and subjectivity. Indianapolis: Bobbs-Merrill

Shapere, D. (1982). Reason, reference, and the quest for knowledge. Philosophy of Science, 40, 485-526

Sharpless, N. E. \& DePinho, R. A. (1999). The ink4a/arf locus and its two gene products. Current Opinion in Genetics and Development, 9, 22-30

Stotz, K. (2006a). Molecular epigenesis: Distributed specificity as a break in the central dogma. History and Philosophy of the Life Sciences, 28, 527-544

Stotz, K. (2006b). With 'genes' like that, who needs an environment? Postgenomics's argument for the 'ontogeny of information'. Philosophy of Science, 73, 905-917

Stotz, K. \& Griffiths, P. E. (2004). Genes: Philosophical analyses put to the test. History and Philosophy of the Life Sciences, 26, 5-28

Sturtevant, A. H. (1915). The behavior of the chromosomes as studied through linkage. Zeitschrift für induktive Abstammungs- und Vererbungslehre, 13, 234-287

Vance, R. E. (1996). Heroic antireductionism and genetics: A tale of one science. Philosophy of Science, 63, S36-S45

Waters, C. K. (1994). Genes made molecular. Philosophy of Science, 61, 163-185

Waters, C. K. (2000). Molecules made biological. Revue Internationale de Philosophie, 4, $539-564$

Waters, C. K. (2004). What concept analysis in philosophy of science should be (and why competing philosophical analyses of gene concepts cannot be tested by polling scientists). History and Philosophy of the Life Sciences, 26, 29-58

Watson, J. D. \& Crick, F. H. C. (1953). Genetical implications of the structure of deoxyribonucleic acid. Nature, 171, 964-967

Weber, M. (2005). Philosophy of experimental biology. Cambridge: Cambridge University Press

Yelin, R., Dahary, D., Sorek, R., Levanon, E. Y., Goldstein, O., Shoshan, A., Diber, A., Biton, S., Tamir, Y., Khosravi, R., Nemzer, S., Pinner, E., Walach, S., Bernstein, J., Savitsky, K. \& Rotman, G. (2003). Widespread occurrence of antisense transcription in the human genome. Nature Biotechnology, 21, 379-386

Zhang, C., Xie, Y. M., Martignetti, J. A., Yeo, T. T., Massa, S. M. \& Longo, F. M. (2003). A candidate chimeric mammalian mRNA transcript is derived from distinct chromosomes and is associated with nonconsensus splice junction motifs. DNA and Cell Biology, 22, 303-315 